\title{
Desingularizations of Schubert varieties in double Grassmannians
}

\author{
Evgeny Smirnov
}

\begin{abstract}
Let $X=\operatorname{Gr}(k, V) \times \operatorname{Gr}(l, V)$ be the direct product of two Grassmann varieties of $k$ - and $l$-planes in a finite-dimensional vector space $V$, and let $B \subset \operatorname{GL}(V)$ be the isotropy group of a complete flag in $V$. We consider $B$-orbits in $X$, which are an analog to Schubert cells in Grassmannians. We describe this set of orbits combinatorially and construct desingularizations for the closures of these orbits, similar to the Bott-Samelson desingularizations for Schubert varieties.
\end{abstract}

\section{Introduction}

Let $V$ be a finite-dimensional vector space over an arbitrary ground field $\mathbb{K}$.

We are interested in describing pairs of subspaces in $V$ of fixed dimensions $k$ and $l$ up to the action of the group $B \subset \mathrm{GL}(V)$ of non-degenerate uppertriangular matrices. So, what we describe is the set of $B$-orbits in the direct product $X=\operatorname{Gr}(k, V) \times \operatorname{Gr}(l, V)$ of two Grassmann varieties. This decomposition is similar to the Schubert decomposition for Grassmannians or to the Ehresmann-Bruhat decomposition for full flag varieties.

The combinatorial description of $B$-orbits in $X$ was given (as a special case of a more general problem) by Magyar, Weyman and Zelevinsky [7] their proof substantially uses quiver theory. The description given below does not refer to these results and is only based some elementary linear algebra. This is a generalization of the description, given in Pin's thesis [8], of orbits in the symmetric space $\mathrm{GL}_{k+l} /\left(\mathrm{GL}_{k} \times \mathrm{GL}_{l}\right)$.

We are also interested in the closures of $B$-orbits in $X$. They can be viewed as analogues of Schubert varieties in Grassmannians. The singularities of Schubert varieties are well-known objects: they admit nice desingularizations, constructed by Bott and Samelson; it is known that Schubert varieties are normal and have rational singularities; their singular loci can be described explicitly. (Good references on this topic are the lecture notes [2] and the textbook [6].) So it is natural to ask the same questions (desingularization, normality, and rationality of singularities) for the closures of $B$-orbits in $X$. In this paper we construct desingularizations of these varieties.

Our interest in this problem is also motivated by the recent paper [3] by G. Bobiński and G. Zwara, where they prove that the singularities of the closures of orbits in representations of type $D$ quivers are equivalent to the singularities of Schubert varieties in double Grassmannians.

The author is grateful to Michel Brion for constant attention to this work, and to Ernest Vinberg and Dmitri Timashev for useful remarks and comments. 


\section{Description of orbits}

\section{$2.1 \quad$ Notation}

Let $V$ be an $n$-dimensional vector space over a field $\mathbb{K}$, and let $k, l<n$ be positive integers. The results of Sec. 2 are valid over an arbitrary ground field; however, in Secs. 3 and 4 we assume $\mathbb{K}$ to be algebraically closed. The direct product $\operatorname{Gr}(k, V) \times$ $\operatorname{Gr}(l, V)$ is denoted by $X$. Usually we do not distinguish between points of $X$ and the corresponding configurations $(U, W)$ of subspaces, where $U, W \subset V, \operatorname{dim} U=k$, and $\operatorname{dim} W=l$.

We fix a Borel subgroup $B$ in $\operatorname{GL}(V)$. Let $V_{\bullet}=\left(V_{1}, \ldots, V_{n}=V\right)$ be the complete flag in $V$ stabilized by $B$.

\subsection{Combinatorial description}

In this section, we introduce some combinatorial objects that parametrize pairs of subspaces up to $B$-action. Namely, pairs of subspaces are parametrized by triples consisting of two Young diagrams contained in the rectangles of size $k \times(n-k)$ and $l \times(n-l)$, respectively, and an involutive permutation in $S_{n}$.

Along with constructing these combinatorial objects, we also construct some "canonical" bases in the spaces $U, W$, and $V$.

Proposition 1. (i). There exist ordered bases $\left(u_{1}, \ldots, u_{k}\right),\left(w_{1}, \ldots, w_{l}\right)$, and $\left(v_{1}, \ldots, v_{n}\right)$ of $U, W$, and $V$, respectively, such that:

- $V_{i}=\left\langle v_{1}, \ldots, v_{i}\right\rangle$ for each $i \in\{1, \ldots, n\}$ (angle brackets stand for the linear span of vectors).

- $u_{i}=v_{\alpha_{i}}$, where $i \in\{1, \ldots, k\}$ and $\left\{\alpha_{1}, \ldots, \alpha_{k}\right\} \subset\{1, \ldots, n\}$.

- The $w_{i}$ are either basis vectors of $V$ or vectors with two-element "support": $w_{i}=v_{\beta_{i}}$ or $w_{i}=v_{\gamma_{i}}+v_{\delta_{i}}$, where $\gamma_{i}>\delta_{i}$; moreover, in the latter case $v_{\gamma_{i}} \in U$ (that is, $\left\{\gamma_{1}, \ldots, \gamma_{r}\right\} \subset\left\{\alpha_{1}, \ldots, \alpha_{k}\right\}$ ).

- All the $\beta_{i}, \gamma_{i}$ and $\delta_{i}$ are distinct; moreover, every $\delta_{i}$ is distinct from every $\alpha_{j}$.

(ii). With the notation of (i), define a permutation $\sigma \in S_{n}$ as the product of all the transpositions $\left(\gamma_{i}, \delta_{i}\right)$. Their supports are disjoint, and so this product does not depend of their order.

Then, for a given pair $(U, W)$, the subsets $\bar{\alpha}=\left\{\alpha_{1}, \ldots, \alpha_{k}\right\}, \bar{\beta}=\left\{\beta_{1}, \ldots, \beta_{l-r}\right\}$, $\bar{\gamma}=\left\{\gamma_{1}, \ldots, \gamma_{r}\right\}$ of $\{1, \ldots, n\}$, and the permutation $\sigma$ are independent of the choice of bases in $U, W$, and $V$.

Proof. (i) We prove this by induction over $n$.

If $n=1$, there is nothing to prove.

The inductive step from $n-1$ to $n$ goes as follows. Take a nonzero vector $v_{1} \in V_{1}$, and consider the following cases:

- $v_{1} \notin U+W$. Let $\bar{V}=V /\left\langle v_{1}\right\rangle$. Let us take the flag $\bar{V}_{\bullet}=\left(\bar{V}_{2} \subset \cdots \subset \bar{V}_{n}\right)$ in this quotient space and consider the images $(\bar{U}, \bar{W})$ of $(U, W)$ under taking the quotient. Both these subspaces are isomorphic to their preimages, $\bar{U} \cong U$ and $\bar{W} \cong W$. Now we apply the induction hypothesis to this configuration as follows. Let us choose ordered bases $\left\{\bar{u}_{1}, \ldots, \bar{u}_{k}\right\},\left\{\bar{w}_{1}, \ldots, \bar{w}_{l}\right\}$, and $\left\{\bar{v}_{1}, \ldots, \bar{v}_{n-1}\right\}$ in $\bar{U}, \bar{W}$, and $\bar{V}$. Then we choose a lift $\imath: \bar{V} \hookrightarrow V$. Now take the preimages of these basis vectors in $V$ in the following way: $u_{i}=\imath\left(\bar{u}_{i}\right)$, $w_{i}=\imath\left(\bar{w}_{i}\right)$, and $v_{i}=\imath\left(\bar{v}_{i-1}\right)$. We obtain the desired triple of bases. 
- $v_{1} \in U, v_{1} \notin W$. Set $u_{1}=v_{1}$ and again apply the induction hypothesis to the quotient $\bar{V}=V /\left\langle v_{1}\right\rangle$ with the flag $\bar{V}_{\bullet}$ and the configuration $(\bar{U}, \bar{W})$. The only difference is that in this case $\operatorname{dim} \bar{U}=\operatorname{dim} U-1$. After that we lift the bases from $\bar{U}, \bar{W}$, and $\bar{V}$ to $V$ in a similar way.

- The case when $v_{1} \notin U$ and $v_{1} \in W$ is similar to the previous one. (We set $w_{1}=v_{1}$.)

- If $v_{1} \in U \cap W$, we set $u_{1}=w_{1}=v_{1}$ and again apply the induction hypothesis.

- The most interesting case is the last one: $v_{1} \in U+W$, but $v_{1} \notin U$ and $v_{1} \notin W$. Consider then the set of vectors $S=\left\{v \mid v \in U, v_{1}+v \in W\right\}$. Since $v_{1}$ belongs to the sum $U+W$, it follows that this set is nonempty. Now let $j$ be the minimum number such that $V_{j}$ contains vectors in $S$, and that $v_{j} \in V_{j} \cap S$. Set $u_{1}=v_{j}$ and $w_{1}=v_{1}+v_{j}$. Now apply the induction hypothesis to the $(n-2)$-dimensional space $\bar{V}=V /\left\langle v_{1}, v_{j}\right\rangle$, the configuration of two subspaces $\bar{U}=U /\left\langle v_{1}, v_{j}\right\rangle, \bar{W}=W /\left\langle v_{1}, v_{j}\right\rangle$, and the flag

$$
\begin{aligned}
\bar{V}_{\bullet}=\left(V_{2} / V_{1} \subset \cdots \subset V_{j-1} / V_{1}=\right. \\
\left.\quad=V_{j} /\left\langle v_{1}, v_{j}\right\rangle \subset V_{j+1} /\left\langle v_{1}, v_{j}\right\rangle \subset \cdots \subset V_{n} /\left\langle v_{1}, v_{j}\right\rangle\right) .
\end{aligned}
$$

We lift basis vectors from $\bar{V}$ to $V$ as follows:

$$
v_{i}=\imath\left(\bar{v}_{i-1}\right), \text { if } i \in[2, j-1] ; \quad v_{i}=\imath\left(\bar{v}_{i-2}\right) \text { if } i \in[j+1, n],
$$

where, as above, $\imath$ is an embedding of $\bar{V}$ in $V$. We have already defined the vectors $v_{1}$ and $v_{j}$.

(ii) Take a configuration $(U, W)$ and assume that there exist two triples of ordered bases, $\left(\left(u_{1}, \ldots, u_{k}\right),\left(w_{1}, \ldots, w_{l}\right),\left(v_{1}, \ldots, v_{n}\right)\right)$ and $\left(\left(u_{1}^{\prime}, \ldots, u_{k}^{\prime}\right),\left(w_{1}^{\prime}, \ldots, w_{l}^{\prime}\right)\right.$, $\left.\left(v_{1}^{\prime}, \ldots, v_{n}^{\prime}\right)\right)$, satisfying the conditions of (i) and such that either the triples of sets $(\bar{\alpha}, \bar{\beta}, \bar{\gamma})$ and $\left(\bar{\alpha}^{\prime}, \bar{\beta}^{\prime}, \bar{\gamma}^{\prime}\right)$ or the permutations $\sigma$ and $\sigma^{\prime}$ corresponding to the first and the second triple of bases, respectively, are not equal.

The set $\bar{\alpha}$ can be described as follows: $i \in \bar{\alpha}$ if and only if $\operatorname{dim} U \cap V_{i}>$ $\operatorname{dim} U \cap V_{i-1}$. This means that $\bar{\alpha}=\bar{\alpha}^{\prime}$.

By the same argument, we can prove that $\bar{\beta} \cup \bar{\gamma}=\bar{\beta}^{\prime} \cup \bar{\gamma}^{\prime}$.

Now let us prove that $\sigma=\sigma^{\prime}$. This will complete the proof, since $\bar{\beta}=\{j \in$ $\bar{\beta} \cup \bar{\gamma} \mid \sigma(j)=j\}$.

Let $j$ be the minimum number in $\bar{\beta} \cup \bar{\gamma}$ such that $\sigma(j) \neq \sigma^{\prime}(j)$. Suppose that $\sigma(j)<\sigma^{\prime}(j)$. Two cases may occur:

a) $i:=\sigma^{\prime}(j) \neq j$. First, observe that $i \notin \bar{\alpha}$. Consider the subspace

$$
\begin{gathered}
\tilde{V}=\left(U \cap V_{j}\right)+V_{i-1}=\left\langle v_{s}, v_{\alpha_{i}} \mid s \leq i-1, \alpha_{i} \in \bar{\alpha} \cup[i, j]\right\rangle= \\
=\left\langle v_{s}^{\prime}, v_{\alpha_{i}}^{\prime} \mid s \leq i-1, \alpha_{i} \in \bar{\alpha}^{\prime} \cup[i, j]\right\rangle .
\end{gathered}
$$

Let $R=\{r \in \bar{\beta} \cup \bar{\gamma} \mid r, \sigma(r) \in[1, i-1] \cup(\bar{\alpha} \cap[i, j])\}$ and $R^{\prime}=\{r \in \bar{\beta} \cup \bar{\gamma} \mid$ $\left.r, \sigma^{\prime}(r) \in[1, i-1] \cup(\bar{\alpha} \cap[i, j])\right\}$. One can readily see that

$$
\operatorname{dim} \tilde{V} \cap W=\# R=\# R^{\prime} .
$$

But $\sigma(r)=\sigma^{\prime}(r)$ for all $r \in[1, j-1]$, and $j$ belongs to $R$ and does not belong to $R^{\prime}$. That means that the cardinalities of these two sets are different, that gives us the desired contradiction.

b) If $\sigma^{\prime}(j)=j$, set $i=\sigma(j)$, and proceed as in a). 
Now introduce a combinatorial construction that parametrizes configuration types. Namely, given a configuration, we will construct a pair of Young diagrams with some boxes marked.

Suppose we have a configuration $(U, W)$. Take the sets $\bar{\alpha}, \bar{\beta}, \bar{\gamma}$, and the involution $\sigma$ corresponding to this configuration, as described in Prop. 1. Consider a rectangle of size $k \times(n-k)$ and construct a path from its bottom-left to top-right corner such that each $j$ th step is vertical if $j$ belongs to $\bar{\alpha}$ (that is, $v_{j}$ is equal to some $u_{i}$ ) and horizontal otherwise. This path bounds (from below) the first Young diagram.

The second diagram is contained in a rectangle of size $l \times(n-l)$. Again, we construct a path bounding it. Let the $j$ th step of this path be vertical if $j \in \bar{\beta} \cup \bar{\gamma}$ and horizontal otherwise.

If $j \in \bar{\gamma}$, then the $\sigma(j)$ th step of this path is horizontal. This also means that the $j$ th and $\sigma(j)$ th steps of the path bounding the first diagram are vertical and horizontal, respectively. In each diagram, take the box located above the $\sigma(j)$ th step and to the left of the $j$ th step and put a dot into each of these boxes.

We refer to this pair of diagrams with dots as a marked pair.

Example. Let $n=9, k=4$, and $l=3$. Suppose that $\bar{\alpha}=\{3,5,6,9\}, \bar{\beta}=\{2,5\}$, $\bar{\gamma}=\{9\}, \sigma=(7,9)$. Then the corresponding marked pair of diagrams is as follows:
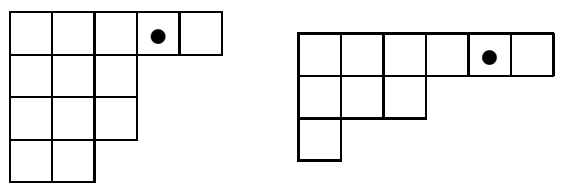

Remark. Note that the constructed diagrams (without dots) are the same as the diagrams that correspond to the Schubert cells containing the points $U \in \operatorname{Gr}(k, V)$ and $W \in \operatorname{Gr}(l, V)$. (The correspondence between Schubert cells and Young diagrams is described, for example, in the textbooks [4] and [6].)

\subsection{Stabilizers and dimensions of orbits}

Now let us find the stabilizer $B_{(U, W)}$ for a given configuration $(U, W)$.

Proposition 2. In the notation of Prop. refbaseschoice, the stabilizer of a configuration $(U, W)$ written in the basis $\left(v_{1}, \ldots, v_{n}\right)$ consists of the upper-triangular matrices $A=\left(a_{i j}\right) \in \mathrm{GL}(n)$ satisfying the following conditions:

(a) $a_{\gamma \gamma}=a_{\sigma(\gamma) \sigma(\gamma)}$ for each $\gamma \in \bar{\gamma}$.

(b) $a_{i \alpha}=0$ for all $\alpha \in \bar{\alpha}$ and $i \notin \bar{\alpha}$.

(c) $a_{j \beta}=0$ for all $\beta \in \bar{\beta}$ and $j \notin \bar{\beta} \cup \bar{\gamma} \cup \sigma(\bar{\gamma})$.

(d) $a_{\gamma \beta}=a_{\sigma(\gamma) \beta}$ for all $\beta \in \bar{\beta}$ and $\gamma \in \bar{\gamma}, \gamma<\beta$.

(e) $a_{j \gamma}=-a_{j \sigma(\gamma)}$ for all $j \notin \bar{\beta} \cup \bar{\gamma} \cup \sigma(\bar{\gamma})$ and $\gamma \in \bar{\gamma}$.

(f) for any $\gamma_{1}, \gamma_{2} \in \bar{\gamma}, \gamma_{1}<\gamma_{2}$, one of the following cases occurs:

- $\sigma\left(\gamma_{2}\right)<\sigma\left(\gamma_{1}\right)<\gamma_{1}<\gamma_{2} ;$ then $a_{\gamma_{1} \gamma_{2}}=a_{\sigma\left(\gamma_{1}\right) \gamma_{2}}=a_{\sigma\left(\gamma_{2}\right) \gamma_{1}}=a_{\sigma\left(\gamma_{1}\right) \sigma\left(\gamma_{2}\right)}=$ 0 .

- $\sigma\left(\gamma_{1}\right)<\sigma\left(\gamma_{2}\right)<\gamma_{1}<\gamma_{2}$; then $a_{\sigma\left(\gamma_{2}\right) \gamma_{1}}=a_{\sigma\left(\gamma_{1}\right) \gamma_{2}}=0, a_{\gamma_{1} \gamma_{2}}=a_{\sigma\left(\gamma_{1}\right) \sigma\left(\gamma_{2}\right)}$.

- $\sigma\left(\gamma_{1}\right)<\gamma_{1}<\sigma\left(\gamma_{2}\right)<\gamma_{2}$; then $a_{\sigma\left(\gamma_{1}\right) \gamma_{2}}=0, a_{\gamma_{1} \gamma_{2}}+a_{\gamma_{1} \sigma\left(\gamma_{2}\right)}=a_{\sigma\left(\gamma_{1}\right) \sigma\left(\gamma_{2}\right)}$.

Corollary 3. The stabilizer of a configuration $(U, W)$ is a semidirect product of a toric and a unipotent part,

$$
B_{(U, W)}=T_{(U, W)} \ltimes U_{(U, W)},
$$


where $T_{(U, W)}$ is the subgroup determined by conditions (a) in the group of nondegenerate diagonal matrices, so that $\operatorname{dim} T_{(U, W)}=n-\# \bar{\gamma}$, and $U_{(U, W)}$ is the subgroup determined by conditions $(b)-(f)$ in the group of unitriangular matrices.

Definition. The codimension of the toric part of the stabilizer is called the rank of a configuration (or its corresponding $B$-orbit):

$$
\operatorname{rk}(U, W):=n-\operatorname{dim} T_{(U, W)}=\# \bar{\gamma} .
$$

Proof of Proposition 2. First, the stabilizer $B_{(U, W)}$ is a subgroup of $B$ and hence consists of upper-triangular matrices.

Next, it preserves the subspace $U=\left\langle v_{\alpha_{1}}, \ldots, v_{\alpha_{k}}\right\rangle$. This means that each transformation $A \in B_{(U, W)}$ takes each $v_{\alpha_{i}}$ to a linear combination of $v_{\alpha_{j}}$, so that all the elements $a_{i \alpha}$ whith $\alpha \in \bar{\alpha}$ and $i \notin \bar{\alpha}$ are zero. (Note that the zeros in $A$ themselves form the Young diagram corresponding to the subspace $U$, rotated $90^{\circ}$ clockwise. This proves, in particular, that the dimension of a Schubert cell in a Grassmannian is equal to the number of boxes in the corresponding Young diagram.)

Thus, the boxes of the first Young diagram are in a one-to-one correspondence with the linear equations defining $B_{U}$ as a subgroup of the group of upper-triangular matrices: the box located above the $i$ th (horizontal) step and to the left of the $j$ th (vertical) step of the corresponding path (denote this box by $(i, j)$ ) corresponds to the equation $a_{i j}=0$.

Likewise, the stabilizer of our configuration preserves the subspace $W$. This gives another set of linear equations on the entries $a_{i j}$, and the number of these equations is equal to the number of boxes in the second diagram of the corresponding marked pair. Again, we establish a one-to-one correspondence between the boxes of this diagram and these equations, denoting boxes as in the previous paragraph. This correspondence is as follows:

- $a_{j \beta}=0$ for all $\beta \in \bar{\beta}$ and $j \notin \bar{\beta} \cup \bar{\gamma} \cup \sigma(\bar{\gamma}), j<\beta$. The corresponding box is $(j, \beta)$.

- $a_{j \gamma}=-a_{j \sigma(\gamma)}$ for all $j \notin \bar{\beta} \cup \bar{\gamma} \cup \sigma(\bar{\gamma})$ and $\gamma \in \bar{\gamma}, j<\gamma$. The corresponding box is $(j, \gamma)$.

- $a_{\sigma(\gamma) \gamma}+a_{\gamma \gamma}-a_{\sigma(\gamma) \sigma(\gamma)}=0$ for all $\gamma \in \bar{\gamma}$. The corresponding box is $(\sigma(\gamma), \gamma)$.

- $a_{\gamma \beta}=a_{\sigma(\gamma) \beta}$ for all $\beta \in \bar{\beta}$ and $\gamma \in \bar{\gamma}, \gamma<\beta$. The corresponding box is $(\sigma(\gamma), \beta)$.

- $a_{\sigma\left(\gamma_{1}\right) \sigma\left(\gamma_{2}\right)}+a_{\sigma\left(\gamma_{1}\right) \gamma_{2}}=a_{\gamma_{1} \sigma\left(\gamma_{2}\right)}+a_{\gamma_{1} \gamma_{2}}$ for any $\gamma_{1}<\gamma_{2}$. This equation corresponds to the box $\left(\sigma\left(\gamma_{1}\right), \gamma_{2}\right)$.

Bringing all these equations together completes the proof of the proposition.

Once we know the stabilizer of a configuration, we can calculate its dimension and hence the dimension of the orbit $B(U, W) \subset X$. Analyzing the equations above, one can find a combinatorial interpretation of dimension in terms of marked pairs of Young diagrams.

To do this, we have to introduce one more combinatorial notion. Suppose we have two rectangles of size $k \times(n-k)$ and $l \times(n-l)$, respectively, and a path in each of these rectangles bounding a Young diagram. (Both paths are of length $n$.) Consider the set of all numbers $i$ such that the $i$ th steps in the paths bounding both diagrams are horizontal and take the columns in the diagrams lying above these steps. After that do the same for the pairs of steps that are "simultaneously vertical" and take the rows to the left of these steps. 
The intersection of columns and rows we have taken is a Young diagram as well. We refer to it as a common diagram corresponding to the given pair of diagrams.

Example. The pair $\left(Y_{1}, Y_{2}\right)$ of Young diagrams
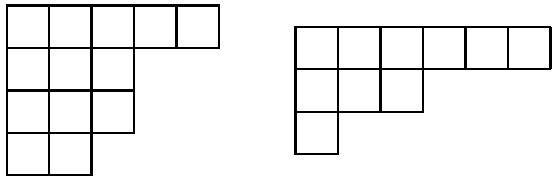

has the following common diagram $Y_{\text {com }}$ :

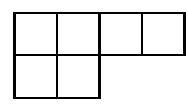

By our construction of marked pairs, dots can only be contained in boxes of the common diagram of a marked pair.

Corollary 4. Let $(U, W)$ be a configuration of subspaces, and let $\left(Y_{1}, Y_{2}\right)$ be the corresponding marked pair of Young diagrams, with dots in some boxes of the common diagram $Y_{\text {com }}$.

Consider the diagram $Y_{\text {com }}$. For a given box, take the set formed by all the boxes in the same row above it, all the boxes in the same row to the left of it, and this box itself. Such set is said to be an inward hook with vertex in the initial box. Now take all its boxes with dots in $Y_{\text {com }}$ and consider all inward hooks with vertices in these boxes. Let $H$ be the set of boxes that belong to at least one of these inward hooks. Then the dimension of the $B$-orbit of $(U, W)$ is equal to

$$
\operatorname{dim} B(U, W)=\# Y_{1}+\# Y_{2}-\# Y_{\text {com }}+\# H,
$$

where $\# Y$ is the number of boxes in $Y$.

Remark. \#H is equal to the total number of boxes contained in all hooks, rather than the sum lengths of all hooks. This means that a box included into two hooks should be counted once, not twice.

Proof. In the proof of Prop. 2, we deal with two systems of linear equations on the matrix entries $a_{i j}$. They correspond to the stabilizers of the subspaces $U$ and $W$ and consist of $\# Y_{1}$ and $\# Y_{2}$ equations, respectively. One can readily see that the equations corresponding to the box $(i, j)$ coincide in both systems if the box $(i, j)$ of the common diagram does not belong to any hook, and also that the system of equations obtained as the union of these two systems is linearly independent. Thus, the codimension of $B_{(U, W)}$ in $B$ (that is, the dimension of $B(U, W)$ ) is equal to $\# Y_{1}+\# Y_{2}-\# Y_{\text {com }}+\# H$.

Example. Let the common diagram for a marked pair be as follows:

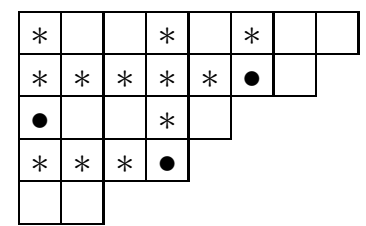

Then $\# Y_{\text {com }}=26$ and $\# H=15$ (Here $H$ consists of all nonempty boxes.)

In particular, the dimension formula allows us to describe the minimal (maximally degenerate) and maximal (open) orbit. The minimal orbit is zero-dimensional and corresponds to $Y_{1}=Y_{2}=\varnothing$. It is the point $\left(\left\langle v_{1}, \ldots, v_{k}\right\rangle,\left\langle v_{1}, \ldots, v_{l}\right\rangle\right) \in X$. 
Both Young diagrams corresponding to the maximal orbit are rectangular, of size $k \times(n-k)$ and $l \times(n-l)$, respectively. Their common diagram is also a rectangle of size $\min \{k, l\} \times(n-\max \{k, l\})$, with dots on the diagonal issuing from the bottom-right corner.

ExAmPle. For $n=8, k=3$, and $l=4$, the combinatorial data corresponding to the maximal orbit are as follows:

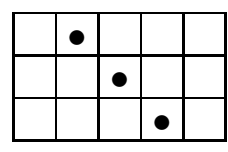

$Y_{1}$

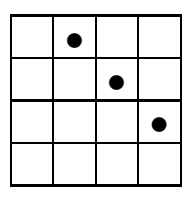

$Y_{2}$

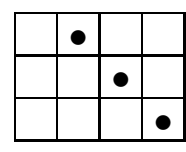

$Y_{\text {com }}$

\subsection{Decomposition of $X$ into a union of GL( $V)$-orbits}

The $\operatorname{GL}(V)$-orbits in $X$ have a much simpler description; they are parametrized by one nonnegative integer, namely, by the dimension of the intersection $U \cap W$. For this number (denote it by $i$ ) we have the inequality

$$
\max \{0, k+l-n\} \leq i \leq \min \{k, l\} .
$$

Denote the corresponding $\mathrm{GL}(V)$-orbit by $X_{i}$ :

$$
X=\bigsqcup_{i \in\{\max (0, k+l-n), \ldots, \min (k, l)\}} X_{i} .
$$

It follows from our construction of the combinatorial data corresponding to a $B$-orbit that $\operatorname{dim}(U \cap W)=\#(\bar{\alpha} \cap \bar{\beta})$.

\section{Weak order on the set of orbits}

In the previous section, we have described the set of $B$-orbits in $\operatorname{Gr}(k, V) \times \operatorname{Gr}(l, V)$. There exist several partial order structures on this set. The first, most natural one is defined as follows:

Definition. Let $\mathcal{O}$ and $\mathcal{O}^{\prime}$ be two $B$-orbits in $\operatorname{Gr}(k, V) \times \operatorname{Gr}(l, V)$. We say that $\mathcal{O}$ is less than or equal to $\mathcal{O}^{\prime}$ with respect to the strong (or topological) order if and only if $\mathcal{O} \subset \overline{\mathcal{O}}^{\prime}$. (Here and further, a bar stands for the Zariski closure of a set). Notation: $\mathcal{O} \leq \mathcal{O}^{\prime}$.

There exists another order on this set, usually called the weak order. Here the notation is taken from [1].

Let $W$ be the Weyl group for $\mathrm{GL}(n)$, and let $\Delta$ be the corresponding root system. Denote the simple reflections by $s_{1}, \ldots, s_{n-1}$, and the corresponding simple roots by $\alpha_{1}, \ldots, \alpha_{n-1}$. Let $P_{i}=B \cup B s_{i} B$ be the minimal parabolic subgroup in $\operatorname{GL}(V)$ corresponding to the simple root $\alpha_{i}$.

We say that $\alpha_{i}$ raises an orbit $\mathcal{O}$ to $\mathcal{O}^{\prime}$, if $\overline{\mathcal{O}^{\prime}}=P_{i} \overline{\mathcal{O}} \neq \overline{\mathcal{O}}$. In this case, $\operatorname{dim} \mathcal{O}^{\prime}=\operatorname{dim} \mathcal{O}+1$. This notion allows us to define the weak order.

Definition. An orbit $\mathcal{O}$ is said to be less than or equal to $\mathcal{O}^{\prime}$ with respect to the weak order (notation: $\mathcal{O} \preceq \mathcal{O}^{\prime}$ ) if $\overline{\mathcal{O}}^{\prime}$ can be obtained as the result of several consecutive raisings of $\overline{\mathcal{O}}$ by minimal parabolic subgroups:

$$
\mathcal{O} \preceq \mathcal{O}^{\prime} \quad \Leftrightarrow \quad \exists\left(i_{1}, \ldots, i_{r}\right): \overline{\mathcal{O}}^{\prime}=P_{i_{r}} \ldots P_{i_{1}} \overline{\mathcal{O}} .
$$


Let us represent this relation of order by an oriented graph. Consider a graph $\Gamma(X)$ with vertices indexed by $B$-orbits in $X$. Join $\mathcal{O}$ and $\mathcal{O}^{\prime}$ with an edge labeled by $i$ and directed to $\mathcal{O}^{\prime}$, if $P_{i}$ raises $\mathcal{O}$ to $\mathcal{O}^{\prime}$.

It is clear that each connected component of $\Gamma(X)$ consists of $B$-orbits contained in a same $\mathrm{GL}(V)$-orbit $X_{i}$ and has a unique maximal element, namely, the $B$-orbit that is open in $X_{i}$.

Our next aim is to describe minimal elements with respect to the weak order in each connected component.

\subsection{Combinatorial description of minimal parabolic sub- group action}

Consider an orbit $\mathcal{O}$ and the corresponding combinatorial data: the sets $\bar{\alpha}, \bar{\beta}$ and $\bar{\gamma}$ and the involution $\sigma \in S_{n}$. Let a minimal parabolic subgroup $P_{i}=B \cup B s_{i} B$ raise the orbit $\mathcal{O}$ to an orbit $\mathcal{O}^{\prime} \neq \mathcal{O}$. Now we shall describe the combinatorial data $\left(\bar{\alpha}^{\prime}\right.$, $\left.\bar{\beta}^{\prime}, \bar{\gamma}^{\prime}, \sigma^{\prime}\right)$ of $\mathcal{O}^{\prime}$.

Denote the transposition $(i, i+1) \in S_{n}$ by $\tau_{i}$.

The following cases may occur:

1. Suppose that

$$
i \in \bar{\alpha}, \quad i \notin \bar{\beta}, \quad i+1 \notin \bar{\alpha}, \quad i+1 \in \bar{\beta},
$$

or, on the opposite,

$$
i \notin \bar{\alpha}, \quad i \in \bar{\beta}, \quad i+1 \in \bar{\alpha}, \quad i+1 \notin \bar{\beta} .
$$

These two cases correspond to two orbits that can be raised to $\mathcal{O}^{\prime}$ by $P_{i}$. In this case, the new combinatorial data for $\mathcal{O}^{\prime}$ is given as follows:

$$
\begin{aligned}
\bar{\alpha}^{\prime} & =\bar{\alpha} \cup\{i+1\} \backslash\{i\} ; \\
\bar{\beta}^{\prime} & =\bar{\beta} \backslash\{i, i+1\} ; \\
\bar{\gamma}^{\prime} & =\bar{\gamma} \cup\{i+1\} \\
\sigma^{\prime} & =\sigma \cdot \tau_{i} .
\end{aligned}
$$

Note that $\operatorname{rk} \mathcal{O}^{\prime}=\operatorname{rk} \mathcal{O}+1$ and $\operatorname{dim} \mathcal{O}^{\prime}=\operatorname{dim} \mathcal{O}+1$.

In the language of marked pairs of diagrams, this is represented as follows. If the $i$ th and the $i+1$ th steps of the path bounding the first diagram form an indentation (i.e., the $i$ th step is vertical and the $(i+1)$ st one is horizontal) and the corresponding intervals of the second diagram form a spike (or, on the opposite, we have a spike in the first diagram and an indentation in the second), then both these pairs of steps are replaced by spikes bounding a marked box.

Example. Apply the minimal parabolic subgroup $P_{2}$ to the orbit $\mathcal{O} \subset$ $\operatorname{Gr}(3,7) \times \operatorname{Gr}(4,7)$ defined by the marked pair
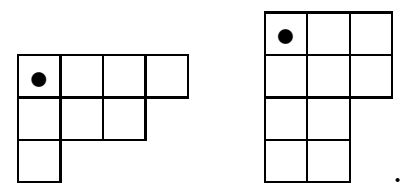

The orbit $\mathcal{O}^{\prime}$ obtained as the result of this raising is defined by the marked pair
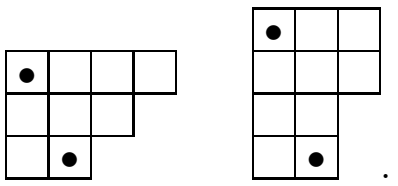
2. In all other cases, $\bar{\alpha}^{\prime}=\tau_{i}(\bar{\alpha}), \bar{\beta}^{\prime}=\tau_{i}(\bar{\beta})$, and $\bar{\gamma}^{\prime}=\tau_{i}(\bar{\gamma})$, and the permutation $\tilde{\sigma}$ is the result of the conjugation of $\sigma$ by $\tau_{i}$ :

$$
\tilde{\sigma}=\tau_{i} \sigma \tau_{i} .
$$

The ranks of these orbits are equal, $\operatorname{rk} \mathcal{O}^{\prime}=\operatorname{rk} \mathcal{O}$.

\subsection{Minimal orbits}

Lemma 5. All minimal B-orbits with respect to the weak order in a given $\mathrm{GL}(V)$ orbit have rank 0.

Proof. Assume the converse. Let $\mathcal{O}$ be a minimal orbit with nonzero rank, and let $(\bar{\alpha}, \bar{\beta}, \bar{\gamma}, \sigma)$ be the corresponding combinatorial data such that $\sigma \neq I d$. Let $p \in \bar{\gamma}$ and $p^{\prime}=\sigma(p)$. Without loss of generality we can suppose that there is no other $q \in \bar{\gamma}$ such that $p<q<\sigma\left(q^{\prime}\right)<p^{\prime}$.

Let $C_{1}$ denote the set of spikes in the first diagram between $p$ th and $p^{\prime}$ th steps, that is, the set of indices $i, p \leq i<p^{\prime}$, such that the $i$ th step in the first diagram is horizontal and the $(i+1)$ st is vertical. Similarly, let $D_{1}$ denote the set of indentations, that is, the set of $i, p \leq i<p^{\prime}$, such that the $i$ th step is vertical and the $(i+1)$ st is horizontal.

Denote the similar sets for the second diagram by $C_{2}$ and $D_{2}$. Note that $\# C_{1}=$ $\# D_{1}+1$ and $\# C_{2}=\# D_{2}+1$, since in both diagrams the $p$ th steps are horizontal and the $p^{\prime}$ th steps are vertical.

Now take a $j \in\left(C_{1} \backslash D_{2}\right) \cup\left(C_{2} \backslash D_{1}\right)$. Using argument in 3.1, we can show that there exists an orbit $\mathcal{O}^{\prime}$ such that $\overline{\mathcal{O}}=P_{j} \overline{\mathcal{O}}^{\prime}$. We describe the combinatorial data for this orbit.

If the permutation $\sigma$ contains the transposition $(j, j+1)$, then the combinatorial data for $\mathcal{O}^{\prime}$ is as follows:

$$
\begin{aligned}
\bar{\alpha}^{\prime} & =\bar{\alpha} \cup\{j\} \backslash\{j+1\} \\
\bar{\beta}^{\prime} & =\bar{\beta} \cup\{j\} \\
\bar{\gamma}^{\prime} & =\bar{\gamma} \backslash\{j+1\} \\
\sigma^{\prime} & =\sigma \cdot \tau_{j} .
\end{aligned}
$$

Otherwise, $\bar{\alpha}^{\prime}=\tau_{j}(\bar{\alpha}), \bar{\beta}^{\prime}=\tau_{j}(\bar{\beta}), \bar{\gamma}^{\prime}=\tau_{j}(\bar{\gamma})$, and $\sigma^{\prime}=\tau_{j} \sigma \tau_{j}$.

The calculation of the dimensions shows that $\operatorname{dim} \mathcal{O}^{\prime}=\operatorname{dim} \mathcal{O}-1$.

To complete the proof, it remains to show that the set $\left(C_{1} \backslash D_{2}\right) \cup\left(C_{2} \backslash D_{1}\right)$ is nonempty:

$$
\begin{aligned}
\#\left(\left(C_{1} \backslash D_{2}\right) \cup\left(C_{2} \backslash D_{1}\right)\right) \geq \max & \left(\#\left(C_{1} \backslash D_{2}\right), \#\left(C_{2} \backslash D_{1}\right)\right) \geq \\
& \geq \max \left(\# C_{1}-\# C_{2}+1, \# C_{2}-\# C_{1}+1\right) \geq 1 .
\end{aligned}
$$

After that we can find all the minimal orbits in $X_{d}$. One can readily see that each minimal orbit has the following combinatorial data:

$$
\begin{aligned}
\bar{\alpha} \cup \bar{\beta} & =\{1, \ldots, k+l-d\} \\
\bar{\alpha} \cap \bar{\beta} & =\{1, \ldots, d\} \\
\bar{\gamma} & =\varnothing \\
\sigma & =I d .
\end{aligned}
$$

The dimensions of all minimal orbits in $X_{d}$ are equal to $(k-d)(l-d)$. In particular, this means that they all are closed in $X_{d}$, since $X_{d}$ does not contain any orbits of 
lower dimension. They correspond to the decompositions of the set $\{d+1, \ldots, k+$ $l-d\}$ into two parts, $\bar{\alpha} \backslash \bar{\beta}$ and $\bar{\beta} \backslash \bar{\alpha}$, and so their number is equal to $\left(\begin{array}{c}k+l-2 d \\ k-d\end{array}\right)$.

Also note that the pair of Young diagrams that corresponds to a minimal orbit is complementary: one can put these two diagrams together so that they fill a rectangle of size $(k-d) \times(l-d)$.

The common diagram corresponding to each of these pairs of diagrams is empty. This means that all minimal orbits are stable under the $(B \times B)$-action, that is, they are direct products of two Schubert cells in two Grassmannians.

These results can be summarized as the following theorem.

Theorem 6. Each $X_{d}$, where $d \in\{\max (k+l-n, 0), \ldots, \min (k, l)\}$, contains $\left(\begin{array}{c}k+l-2 d \\ k-d\end{array}\right)$ minimal orbits. All these orbits are closed in $X_{d}$ and have dimension $(k-d)(l-d)$. They are direct products of Schubert cells.

\section{Desingularizations of the orbit closures}

In this section, we construct desingularizations for the closures of $B$-orbits in $X$.

Given a minimal parabolic subgroup $P_{i}$ and the closure $\overline{\mathcal{O}}$ of an orbit, consider the morphism

$$
\begin{gathered}
F_{i}: P_{i} \times{ }^{B} \overline{\mathcal{O}} \rightarrow P_{i} \overline{\mathcal{O}}, \\
(p, x) \mapsto p x .
\end{gathered}
$$

Suppose that $\overline{\mathcal{O}} \neq P_{i} \overline{\mathcal{O}}$. Knop [5] and Richardson-Springer [9] showed that the following three cases can occur:

- Type $\mathrm{U}: P_{i} \mathcal{O}=\mathcal{O}^{\prime} \sqcup \mathcal{O}$, and $F_{i}$ is birational.

- Type $\mathrm{N}: P_{i} \mathcal{O}=\mathcal{O}^{\prime} \sqcup \mathcal{O}$, and $F_{i}$ is of degree 2 .

- Type T: $P_{i} \mathcal{O}=\mathcal{O}^{\prime} \sqcup \mathcal{O} \sqcup \mathcal{O}^{\prime \prime}$, and $F_{i}$ is birational. In this case, $\operatorname{dim} \mathcal{O}^{\prime \prime}=\operatorname{dim} \mathcal{O}$.

It turns out that type case $\mathrm{N}$ never occurs in our situation.

Proposition 7. Let $\mathcal{O}$ be a $B$-orbit in $X$, and let $P_{i}$ be a minimal parabolic subgroup raising this orbit. Then the map $F_{i}: P_{i} \times{ }^{B} \mathcal{O} \rightarrow P_{i} \mathcal{O}$ is birational.

Proof. Choose the canonical representative $x \in \mathcal{O}$ as in Prop. 1. A straightforward calculation shows that the isotropy group of $x$ in $P_{i}$ is equal to the stabilizer of $x$ in $B$, described in Prop. 2, This implies the birationality of $F_{i}$.

Remark. The two remaining cases correspond to the two possible "raisings" described in the subsection 3.1? (T) corresponds to item 1, and (U) corresponds to item 2. In the first case, the rank of the orbit is increased by one, and in the second case, it does not change. Thus, the weak order is compatible with the rank function: if $\mathcal{O} \preceq \mathcal{O}^{\prime}$, then $\mathrm{rk} \mathcal{O} \leq \mathrm{rk} \mathcal{O}^{\prime}$. This is true in general for spherical varieties (cf., for instance, [1]). Note that the strong order is not compatible with the rank function.

Proposition 17, together with Theorem 6, allows us to construct desingularizations for $\overline{\mathcal{O}}$ 's similar to Bott-Samelson desingularizations of Schubert varieties in Grassmannians.

Given an orbit $\mathcal{O}$, consider a minimal orbit $\mathcal{O}_{\text {min }}$ that is less than or equal to than $\mathcal{O}$ with respect to the weak order. This means that there exists a sequence $\left(P_{i_{1}}, \ldots, P_{i_{r}}\right)$ of minimal parabolic subgroups such that

$$
\overline{\mathcal{O}}=P_{i_{r}} \ldots P_{i_{1}} \overline{\mathcal{O}}_{m i n}
$$


Consider the map

$$
\begin{gathered}
F: P_{i_{r}} \times{ }^{B} \ldots \times{ }^{B} P_{i_{1}} \times{ }^{B} \overline{\mathcal{O}}_{\text {min }} \rightarrow \overline{\mathcal{O}}, \\
F:\left(p_{i_{r}}, \ldots, p_{i_{1}}, x\right) \mapsto p_{i_{r}} \ldots p_{i_{1}} x .
\end{gathered}
$$

By Proposition 7, it is birational. But this is not yet a desingularization, because $\overline{\mathcal{O}}_{\text {min }}$ can be singular.

The second step of desingularization consists in constructing a $B$-equivariant desingularization for $\overline{\mathcal{O}}_{\text {min }}$. We have already proved in Theorem 6 that $\overline{\mathcal{O}}_{\text {min }}$ can be represented as the direct product

$$
\overline{\mathcal{O}}_{\min }=X_{w} \times X_{v}
$$

for some Schubert varieties $X_{w} \subset \operatorname{Gr}(k, V)$ and $X_{v} \subset \operatorname{Gr}(l, V)$.

For $X_{w}$ and $X_{v}$, one can take Bott-Samelson desingularizations

$$
F_{w}: Z_{w} \rightarrow X_{w} \quad \text { and } \quad F_{v}: Z_{v} \rightarrow X_{v} .
$$

(Details can be found, for instance, in [2].) Thus, we obtain the desingularization

$$
F_{w} \times F_{v}: \quad Z_{w} \times Z_{w} \rightarrow X_{w} \times X_{v}=\overline{\mathcal{O}}_{\text {min }} .
$$

Having this, we can combine this map with the map $F$ and obtain the main result of this paper:

Theorem 8. The map

$$
\tilde{F}=F \circ\left(F_{w} \times F_{v}\right): \quad P_{i_{r}} \times{ }^{B} \cdots \times{ }^{B} P_{i_{1}} \times{ }^{B}\left(Z_{w} \times Z_{v}\right) \rightarrow \overline{\mathcal{O}}
$$

is a desingularization of $\overline{\mathcal{O}}$.

Proof. We have already seen that both maps $F$ and $F_{w} \times F_{v}$ are birational morphisms. Since all the considered varieties are projective, these morphisms are proper. The variety $P_{i_{r}} \times{ }^{B} \cdots \times{ }^{B} P_{i_{1}} \times{ }^{B}\left(Z_{w} \times Z_{v}\right)$ is a sequence of homogeneous $B$-bundles with nonsingular fibers, and hence it is nonsingular itself.

\section{References}

[1] M. Brion, On orbit closures of spherical subgroups in flag varieties, Comment. Math. Helv. 76 (2001), 263-299.

[2] M. Brion, Lectures on the geometry of flag varieties, Topics in cohomological studies of algebraic varieties, 33-85, Trends Math., Birkhäuser, Basel, 2005.

[3] G. Bobiński, G. Zwara, Schubert varieties and representations of Dynkin quivers, Colloquium Mathematicum 94 (2002), 285-309.

[4] W. Fulton, Young tableaux with applications to representation theory and geometry, Cambridge Univ. Press, 1997.

[5] F. Knop, On the set of orbits for a Borel subgroup. Comment. Math. Helv., 70 (2), pp. 285-309, 1995.

[6] L. Manivel, Fonctions symétriques, polynômes de Schubert et lieux de dégénérescence, Société Mathématique de France, 1998

[7] P. Magyar, J. Weyman, A. Zelevinsky, Multiple flag varieties of finite type, Adv. Math. 141:1 (1999), 97-118. 
[8] S. Pin, Adhérences d'orbites des sous-groupes de Borel dans les éspaces symétriques, thèse de doctorat, Institut Fourier, Grenoble, 2001. http://www-fourier.ujf-grenoble.fr/THESE/ps/t107.ps.gz

[9] R. W. Richardson, T. A. Springer, The Bruhat order on symmetric varieties, Geom. Dedicata, 35 (1990), 389-436.

Independent University of Moscow, Bolshoi Vlasievskil Per., 11, 119002 Moscow, RUSSIA

Institut Fourier, 100 Rue des Maths, 38400 Saint-Martin d'Hères, France

E-mail address: smirnoff@mccme.ru

Keywords: Grassmannian, spherical variety, desingularization 